\title{
THE ZERO-REMOVING PROPERTY AND LAGRANGE-TYPE INTERPOLATION SERIES
}

\author{
P. E. Fernàndez-Moncada, ${ }^{1}$ A. G. García, ${ }^{1}$ and M. A. Hernández-Medina ${ }^{2}$ \\ ${ }^{1}$ Departamento de Matemáticas, Universidad Carlos III de Madrid, \\ Leganés-Madrid, Spain \\ ${ }^{2}$ Departamento de Matemàtica Aplicada, E.T.S.I.T., U.P.M., Madrid, Spain
}

\begin{abstract}
The classical Kramer sampling theorem, which provides a method for obtaining orthogonal sampling formulas, can be formulated in a more general nonorthogonal setting. In this setting, a challenging problem is to characterize the situations when the obtained nonorthogonal sampling formulas can be expressed as Lagrange-type interpolation series. In this article a necessary and sufficient condition is given in terms of the zero removing property. Roughly speaking, this property concerns the stability of the sampled functions on remoring a finite number of their zeros.
\end{abstract}

Keywords Analytic Kramer kernels; Lagrange-type interpolation series; Zeroremoving property.

AMS Subject Classification 46E22; 42C15; $94 \mathrm{~A} 20$.

\section{STATEMENT OF THE PROBLEM}

The classical Kramer sampling theorem provides a method for obtaining orthogonal sampling theorems $[5,13,15,21]$. The statement of this general result is as follows. Let $K$ be a complex function defined on $D \times I$, where $I \subset \mathbb{R}$ is an interval and $D$ is an open subset of $\mathbb{R}$, and such that for every $t \in D$ the sections $K(\cdot, t)$ are in $\mathscr{L}^{2}(I)$. Assume that there exists a sequence of distinct real numbers $\left\{t_{n}\right\} \subset D$, indexed by a subset of $\mathbb{Z}$, such that $\left\{K\left(x, t_{n}\right)\right\}$ is a complete orthogonal sequence of functions for $\mathscr{L}^{2}(I)$. Then for any $f$ of the form

$$
f(t)=\int_{I} F(x) K(x, t) d x \quad t \in D,
$$

Received I5 February 20II; Revised 28 April 20II; Accepted 4 May $20 \mathrm{II}$.

Address correspondence to A. G. García, Departamento de Matemáticas, Universidad Carlos III de Madrid, Avda. de la Universidad, 30, Leganès-Madrid 2891 I, Spain; E-mail: pagarcia@math.uc3m.es 
where $F \in \mathscr{L}^{2}(I)$, we have

$$
f(t)=\sum_{n} f\left(t_{n}\right) S_{n}(t), \quad t \in D
$$

with

$$
S_{n}(t):=\frac{\int_{I} K(x, t) \overline{K\left(x, t_{n}\right)} d x}{\int_{I}\left|K\left(x, t_{n}\right)\right|^{2} d x} .
$$

The series in (2) converges absolutely and uniformly on subsets of $D$ where $\|K(\cdot, t)\|_{\mathscr{L}^{2}(I)}$ is bounded.

For instance, taking $I=[-\pi, \pi], K(x, t)=\mathrm{e}^{\mathrm{i} t x}$ and $\left\{t_{n}=n\right\}_{n \in \mathbb{Z}}$, we get the well-known Whittaker-Shannon-Kotel'nikov sampling formula

$$
f(t)=\sum_{n=-\infty}^{\infty} f(n) \frac{\sin \pi(t-n)}{\pi(t-n)}, \quad t \in \mathbb{R},
$$

for functions in $L^{2}(\mathbb{R})$ whose Fourier transform has support in $[-\pi, \pi]$.

Now, if we take $I=[0,1], K(x, t)=\sqrt{x t} J_{v}(x t)$ and $\left\{t_{n}\right\}$, the sequence of the positive zeros of the Bessel function $J_{v}$ of $v$ th order with $v>-1$, then

$$
f(t)=\sum_{n} f\left(t_{n}\right) \frac{2 \sqrt{t_{n}} t J_{v}(t)}{J_{v}^{\prime}\left(t_{n}\right)\left(t^{2}-t_{n}^{2}\right)}, \quad t \in \mathbb{R},
$$

for every $f$ of the form $f(t)=\int_{0}^{1} F(x) \sqrt{x t} J_{v}(x t) d x$, where $F \in L^{2}(0,1)$ (see $[13$, p. 83]).

The Kramer sampling theorem has played a very significant role in sampling theory, interpolation theory, signal analysis and, generally, in mathematics (see, e.g., the survey articles [3, 4]).

In [6], an extension of the Kramer sampling theorem has been obtained to the case when the kernel is analytic in the sampling parameter $t \in D \subseteq \mathbb{C}$. Namely, assume that the Kramer kernel $K$ is an entire function for any fixed $x \in I$, and that the function $h(t)=\int_{I}|K(x, t)|^{2} d x$ is locally bounded on $D \subseteq \mathbb{C}$. Then any function $f$ defined by (1) is an entire function, as are all the sampling functions (3).

A straightforward discrete version of Kramer's theorem can be obtained. Namely, let $K(n, z)$ be a kernel such that, as function of $n$, the sequence $\{K(n, z)\} \in \ell^{2}(\mathbb{I})$ for any $z \in D \subseteq \mathbb{C}$, where II is a countable index set. Assume that, for a suitable sequence $\left\{z_{n}\right\} \subset D$, the sequence $\left\{K\left(\cdot, z_{n}\right)\right\}$ is an orthogonal basis for $\ell^{2}(\mathbb{I})$. Then, any function of the form $f(z)=\sum_{n \in \mathbb{I}} c_{n} K(n, z)$, where $\left\{c_{n}\right\} \in \ell^{2}($ II $)$, can be expanded by means of a sampling series like (2) (see [8]). As examples of discrete kernels for which a sampling formula works we can consider discrete kernels 
$K(n, z):=P_{n}(z), n \in \mathbb{N}_{0}:=\mathbb{N} \cup\{0\}$ and $z \in \mathbb{C}$, where $\left\{P_{n}(z)\right\}_{n \in \mathbb{N}_{0}}$ denotes a sequence of orthonormal polynomials associated with an indeterminate Hamburger or Stieltjes moment problem (see $[8,9]$ for the details).

The Kramer sampling theorem has been the cornerstone for a significant mathematical literature of sampling theory associated with differential or difference problems. See, among others, [1, 5, 8, 9, 13, 21] and the references therein.

Thus an abstract analytic formulation of the Kramer sampling theorem raises in a natural way: Let $\mathscr{H}$ be a complex, separable Hilbert space with inner product $\langle\cdot, \cdot\rangle_{\mathscr{H}}$, and let $\left\{x_{n}\right\}_{n=1}^{\infty}$ be a Riesz basis for $\mathscr{H}$. Suppose $K$ is a $\mathscr{H}$-valued function defined on $\mathbb{C}$. For each $x \in \mathscr{H}$, define the function $f_{x}(z)=\langle K(z), x\rangle_{\mathscr{H}}$ on $\mathbb{C}$, and let $\mathscr{H}_{K}$ denote the collection of all such functions $f_{x}$. Furthermore, each element in $\mathscr{H}_{K}$ is an entire function if and only if $K$ is analytic on $\mathbb{C}$. In this setting, an abstract version of the analytic Kramer theorem is obtained assuming the existence of two sequences, $\left\{z_{n}\right\}_{n=1}^{\infty}$ in $\mathbb{C}$ and $\left\{a_{n}\right\}_{n=1}^{\infty}$ in $\mathbb{C} \backslash\{0\}$, such that $K\left(z_{n}\right)=a_{n} x_{n}$ for each $n \in \mathbb{N}$. Namely, for any $f_{x} \in \mathscr{H}_{K}$ we have

$$
f_{x}(z)=\sum_{n=1}^{\infty} f_{x}\left(z_{n}\right) \frac{S_{n}(z)}{a_{n}}, \quad z \in \mathbb{C},
$$

where $S_{n}(z)=\left\langle K(z), y_{n}\right\rangle, n \in \mathbb{N}$, being $\left\{y_{n}\right\}_{n=1}^{\infty}$ the dual Riesz basis of $\left\{x_{n}\right\}_{n=1}^{\infty}$ (see sections 2 and 4 infra for all the details).

A challenging problem is to give a necessary and sufficient condition to ensure that the above sampling formula can be written as a Lagrange-type interpolation series, that is

$$
f_{x}(z)=\sum_{n=1}^{\infty} f_{x}\left(z_{n}\right) \frac{P(z)}{\left(z-z_{n}\right) P\left(z_{n}\right)}, \quad z \in \mathbb{C},
$$

where $P$ denotes an entire function having only simple zeros at all the points of the sequence $\left\{z_{n}\right\}_{n=1}^{\infty}$. Roughly speaking, the aforesaid necessary and sufficient condition concerns the stability of the functions belonging to the space $\mathscr{H}_{K}$ on removing a finite number of their zeros; this is an ubiquitous algebraic property in the mathematical literature (see section 3 infra) and it will be called the zero-removing property along the article.

Let us consider the following toy example: Given a basis $\left\{\mathbf{e}_{1}, \mathbf{e}_{2}\right\}$ in $\mathbb{C}^{2}$, for the kernel $K(z):=z^{2}\left(\mathbf{e}_{2}-\mathbf{e}_{1}\right)+\mathbf{e}_{1}$ consider the corresponding space $\mathscr{H}_{K}$, which coincides with $\left\{a z^{2}+b \mid a, b \in \mathbb{C}\right\}$. Obviously, this space has not the zero-removing property: if we remove a zero from an element in $\mathscr{H}_{K}$ the resulting polynomial does not belong to $\mathscr{H}_{K}$. Besides, the sampling formula $f(z)=f(0)\left(1-z^{2}\right)+f(1) z^{2}$, which holds in $\mathscr{H}_{K}$ cannot be written as a Lagrange interpolation formula. The study of all these topics will be carried out throughout the remaining sections. 


\section{SOME PRELIMINARIES ON THE SPACE $\mathscr{H}_{K}$}

Suppose we are given a separable complex Hilbert space $\mathscr{H}$ and an abstract kernel $K$ which is nothing but a $\mathscr{H}$-valued function on $\mathbb{C}$. Set $f_{x}(z):=\langle K(z), x\rangle_{\mathscr{H}}$ and denote by $\mathscr{H}_{K}$ the collection of all such functions $f_{x}, x \in \mathscr{H}$. It is a reproducing kernel Hilbert space (RKHS) coming from the transforms $K(z), z \in \mathbb{C}$, and corresponding to the reproducing kernel $(z, w) \mapsto\langle K(z), K(w)\rangle_{\mathscr{H}}$. Notice that the mapping $\mathscr{T}$ given by

$$
\mathscr{H} \ni x \stackrel{\mathscr{T}}{\longmapsto} f_{x} \in \mathscr{H}_{K}
$$

is an antilinear mapping from $\mathscr{H}$ onto $\mathscr{H}_{K}$ (henceforth we omit the subscript $x$ for denoting the elements in $\mathscr{H}_{K}$ ). The mapping $\mathscr{T}$ is injective if and only if the set $\{K(z)\}_{z \in \mathbb{C}}$ is a complete set in $\mathscr{H}$. In particular, if there exists a sequence $\left\{z_{n}\right\}_{n=1}^{\infty}$ in $\mathbb{C}$ such that $\left\{K\left(z_{n}\right)\right\}_{n=1}^{\infty}$ is a Riesz basis for $\mathscr{H}$, then $\mathscr{T}$ is an antilinear isometry from $\mathscr{H}$ onto $\mathscr{H}_{K}$. Recall that a Riesz basis in a separable Hilbert space $\mathscr{H}$ is the image of an orthonormal basis by means of a boundedly invertible operator. Any Riesz basis $\left\{x_{n}\right\}_{n=1}^{\infty}$ has a unique biorthonormal (dual) Riesz basis $\left\{y_{n}\right\}_{n=1}^{\infty}$, i.e., $\left\langle x_{n}, y_{m}\right\rangle_{\mathscr{H}}=\delta_{n, m}$, such that the expansions

$$
x=\sum_{n=1}^{\infty}\left\langle x, y_{n}\right\rangle_{\mathscr{K}} x_{n}=\sum_{n=1}^{\infty}\left\langle x, x_{n}\right\rangle_{\mathscr{H}} y_{n}
$$

hold for every $x \in \mathscr{H}$ (see [20] for more details and proofs).

The convergence in the norm $\|\cdot\|_{\mathscr{H}_{K}}$ implies pointwise convergence which is uniform on those subsets of $\mathbb{C}$ where the function $z \mapsto\|K(z)\|_{\mathscr{H}}$ is bounded.

Like in the classical case the following result holds: The space $\mathscr{H}_{K}$ is a RKHS of entire functions if and only if the kernel $K$ is analytic in $\mathbb{C}[19$, p. 266]. Another characterization of the analyticity of the functions in $\mathscr{H}_{K}$ is given in terms of Riesz bases. Suppose that a Riesz basis $\left\{x_{n}\right\}_{n=1}^{\infty}$ for $\mathscr{H}$ is given and let $\left\{y_{n}\right\}_{n=1}^{\infty}$ be its dual Riesz basis; expanding $K(z)$, for each fixed $z \in \mathbb{C}$, with respect to the basis $\left\{x_{n}\right\}_{n=1}^{\infty}$ we obtain

$$
K(z)=\sum_{n=1}^{\infty}\left\langle K(z), y_{n}\right\rangle_{\mathscr{H}} x_{n}
$$

where the coefficients $\left\langle K(z), y_{n}\right\rangle_{\mathscr{H}}$ as functions in $z$ are in $\mathscr{H}_{K}$. The following result holds: The space $\mathscr{H}_{K}$ is a RKHS of entire functions if and only if all the functions

$$
S_{n}(z):=\left\langle K(z), y_{n}\right\rangle_{\mathscr{H}}, \quad z \in \mathbb{C}
$$

are entire and $\|K(\cdot)\|_{\mathscr{H}}$ is bounded on compact sets of $\mathbb{C}$ (see [11]). 


\section{THE ZERO-REMOVING PROPERTY}

In this section, we introduce the zero-removing property for classes of entire functions.

Definition 1 (Zero-Removing Property). A set $\mathscr{A}$ of entire functions has the zero-removing property (ZR property hereafter) if for any $g \in \mathscr{A}$ and any zero $w$ of $g$ the function $g(z) /(z-w)$ belongs to $A$.

The ZR property is ubiquitous in mathematics; for instance, the set $\mathscr{P}_{N}(\mathbb{C})$ of polynomials with complex coefficients of degree less or equal $N$ has the ZR property. Another more involved examples sharing this property are:

- The entire functions in the Pólya class have the ZR property [2, p. 15]. Recall that an entire function $E(z)$ is said to be of Pólya class if it has no zeros in the upper half-plane, if $|E(x-i y)| \leq|E(x+i y)|$ for $y>0$, and if $|E(x+i y)|$ is a nondecreasing function of $y>0$ for each fixed $x$.

- The entire functions in the Paley-Wiener class $P W_{\pi}$ of bandlimited functions to $[-\pi, \pi]$, that is, $P W_{\pi}:=\left\{f \in \mathscr{L}^{2}(\mathbb{R}) \cap C(\mathbb{R}): \operatorname{supp} \hat{f} \subseteq\right.$ $[-\pi, \pi]\}$, where $\hat{f}$ stands for the Fourier transform of $f$, satisfy the ZR property; it follows from the classical Paley-Wiener theorem [20, p. 101], which says that this space can be written as $P W_{\pi}=\{f$ entire function : $\left.|f(z)| \leq A \mathrm{e}^{\pi|z|},\left.f\right|_{\mathbb{R}} \in \mathscr{L}^{2}(\mathbb{R})\right\}$. From this characterization the ZR property immediately comes out.

- In general, de Branges spaces $\mathscr{H}(E)$ with strict de Branges function $E$ have the ZR property [2, p. 52]. Let $E$ be an entire function verifying $|E(x-i y)|<|E(x+i y)|$ for all $y>0$. The de Branges space $\mathscr{H}(E)$ is the set of all entire functions $F$ such that

$$
\|F\|_{E}^{2}:=\int_{-\infty}^{\infty}\left|\frac{F(t)}{E(t)}\right|^{2} d t<\infty,
$$

and such that both ratios $F / E$ and $F^{*} / E$, where $F^{*}(z):=\overline{F(\bar{z})}$, are of bounded type and of non-positive mean type in the upper half-plane.

The structure function or de Branges function $E$ has no zeros in the upper half plane. A de Branges function $E$ is said to be strict if it has no zeros on the real axis. We require that $F / E$ and $F^{*} / E$ be of bounded type and nonpositive mean type in $\mathbb{C}^{+}$. A function is of bounded type if it can be written as a quotient of two bounded analytic functions in $\mathbb{C}^{+}$ and it is of nonpositive mean type if it grows no faster than $e^{\varepsilon y}$ for each $\varepsilon>0$ as $y \rightarrow \infty$ on the positive imaginary axis $\{\mathrm{i} y: y>0\}$. Note that the Paley-Wiener space $P W_{\pi}$ is a de Branges space for the structure function $E_{\pi}(z)=\exp (-\mathbf{i} \pi z)$. 
Assume that the space $\mathscr{H}_{K}$ in section 2 comes from a polynomial kernel $K$ with coefficients in $\mathscr{H}$; concerning the ZR property in $\mathscr{H}_{K}$, the following result holds:

Theorem 1. The space $\mathscr{H}_{K}$ associated with a polynomial kernel $K(z):=$ $\sum_{n=0}^{N} p_{n} z^{n}$, where $p_{n} \in \mathscr{H}$ and $p_{N} \neq 0$, has the $Z R$ property if and only if the set $\left\{p_{0}, p_{1}, \ldots, p_{N}\right\}$ is linearly independent in $\mathscr{H}$.

Proof. Consider $f(z)=a_{N} z^{N}+\cdots+a_{1} z+a_{0} \in \mathscr{H}_{K}$ with $a_{N} \neq 0$; there exists $x \in \mathscr{H}$ such that $f(z)=\langle K(z), x\rangle$ and, consequently, $a_{j}=\left\langle p_{j}, x\right\rangle$ for $j=0,1, \ldots, N$. If the space $\mathscr{H}_{K}$ has the ZR property and $\alpha_{0}, \alpha_{1}, \ldots, \alpha_{N}$ are the roots of the polynomial $f$ then the constant $a_{N}$ and the polynomials $a_{N}\left(z-\alpha_{N}\right), a_{N}\left(z-\alpha_{N}\right)\left(z-\alpha_{N-1}\right), \ldots, a_{N}\left(z-\alpha_{N}\right)\left(z-\alpha_{N-1}\right) \cdots\left(z-\alpha_{1}\right)$

belong to $\mathscr{H}_{K}$. Let $b_{0}, b_{1}, \ldots, b_{N} \in \mathbb{C}$ such that

$$
b_{N} p_{N}+b_{N-1} p_{N-1}+\cdots+b_{0} p_{0}=0 .
$$

The vector $\left(b_{N}, \ldots, b_{0}\right)$ is orthogonal in $\mathbb{C}^{N+1}$ to any vector $\left(c_{N}, \ldots, c_{0}\right) \in$ $\mathbb{C}^{N+1}$ with $c_{N} z^{N}+\cdots+c_{0} \in \mathscr{H}_{K}$. As a consequence, since $a_{N} \in \mathscr{H}_{K}, b_{0} a_{N}=0$, which implies that $b_{0}=0$. Analogously, since $a_{N}\left(z-\alpha_{N}\right)$ belongs to $\mathscr{H}_{K}$ we have that $a_{N} b_{1}-\left(a_{N} \alpha_{N}\right) b_{0}=0$ and consequently $b_{1}=0$. Proceeding iteratively it is straightforward to obtain that $b_{2}=\cdots=b_{N-1}=0$; finally, from (6) we conclude that $b_{N}=0$.

Now suppose that the set $\left\{p_{0}, p_{1}, \ldots, p_{N}\right\}$ is linearly independent in $\mathscr{H}$. In this case, the mapping $\Phi: \mathscr{H} \rightarrow \mathbb{C}^{N+1}$ given by $\Phi(x)=$ $\left(\left\langle p_{0}, x\right\rangle, \ldots,\left\langle p_{N}, x\right\rangle\right)$ is surjective. As a consequence, any complex polynomial of degree less than or equal to $N$ belongs to $\mathscr{H}_{K}$. Let $f(z)=a_{N} z^{n}+\cdots+a_{1} z+a_{0} \in \mathscr{H}_{K}$ and let $w \in \mathbb{C}$ be a root of $f$. Hence, $f(z) /(z-w)=c_{0}+c_{1} z+\cdots+c_{N-1} z^{N-1}$ is a polynomial of degree less than or equal to $N-1$. Since $\Phi$ is onto there exists $x \in \mathscr{H}$ such that $\Phi(x)=\left(c_{0}, c_{1}, \ldots, c_{N-1}, 0\right)$. From the definition of $\Phi$, we conclude that $f(z) /(z-w)=\langle K(z), x\rangle$, that is, the function $f(z) /(z-w) \in \mathscr{H}_{K}$.

Giving a necessary and sufficient for a general analytic kernel $K$ remains as an open problem. It is worth to mention that a straightforward application of Cauchy-Schwarz inequality shows that entire functions in $\mathscr{H}_{K}$ inherit the finite order and the type of the vector-valued entire function $K$ provided it has finite order.

As examples of spaces $\mathscr{H}_{K}$ where the ZR property does not hold let us mention the following:

- Consider the spaces $\mathscr{H}_{K_{i}}, i=1,2$, associated with the analytic kernels $K_{i}$ : $\mathbb{C} \rightarrow L^{2}[0, \pi]$ defined by $K_{1}(z)[x]:=\sin z x$ and $K_{2}(z)[x]:=\cos z x$. The space $\mathscr{H}_{K_{1}}$ corresponds to the space of odd bandlimited functions in $P W_{\pi}$ 
while $\mathscr{H}_{K_{2}}$ corresponds to the space of even bandlimited functions in $P W_{\pi}$. It is clear that the ZR property does not hold in these spaces.

- Let $K: \mathbb{C} \rightarrow \mathscr{H}$ be an analytic kernel such that $K\left(z_{0}\right)=0$ for some $z_{0} \in \mathbb{C}$. Then all the functions in the associated space $\mathscr{H}_{K}$ have a zero at $z_{0}$ and the ZR property does not hold in $\mathscr{H}_{K}$. Indeed, let $f$ be a nonzero entire function in $\mathscr{H}_{K}$ and let $r$ denote the order of its zero $z_{0}$. The function $f(z) /\left(z-z_{0}\right)^{r}$ is not in $\mathscr{H}_{K}$ since it does not vanish at $z_{0}$.

- A little more sophisticated example is the following: For $m \geq 2$ let $K_{m}: \mathbb{C} \rightarrow L^{2}[-\pi, \pi]$ be defined as $K_{m}(z)=\frac{1}{\sqrt{2 \pi}} \mathrm{e}^{\mathrm{i} z^{m}}, \in L^{2}[-\pi, \pi]$. It is straightforward to show that $K_{m}$ is an analytic kernel; the corresponding space $\mathscr{H}_{K_{m}}$ does not have the ZR property. Indeed, expanding $K_{m}(z)$ as power series around the origin we obtain

$$
\left[K_{m}(z)\right](x)=\sum_{k=0}^{\infty} \frac{(\mathrm{i} x)^{k} z^{m k}}{k !}=1+\mathrm{i} x z^{m}-\frac{x^{2} z^{2 m}}{2 !}-\mathrm{i} \frac{x^{3} z^{3 m}}{3 !}+\cdots .
$$

Thus, for any function $f(z)=\left\langle K_{m}(z), F\right\rangle$ with $F \in L^{2}[-\pi, \pi]$ we have

$$
f(z)=\sum_{k=0}^{\infty} c_{k} z^{m k}
$$

where $c_{k}=\left\langle(\mathbf{i} x)^{k} / k !, F\right\rangle, k=0,1, \ldots$ Let $G \in L^{2}[-\pi, \pi] \backslash\{0\}$ be such that $G$ is orthogonal to $K(0)$ and let $g(z)=\left\langle K_{m}(z), G\right\rangle$. Since $\langle K(0), G\rangle=0$ we have $g(0)=0$. Hence, the Taylor expansion of $g(z) / z$ around the origin has the form

$$
\frac{g(z)}{z}=d_{1} z^{m-1}+d_{2} z^{2 m-1}+\cdots
$$

where $d_{k}=\left\langle(\mathrm{i} x)^{k} / k !, G\right\rangle, k=1,2, \ldots$ Since $G$ is not the zero function the function $g(z) / z$ does not belong to $\mathscr{H}_{K_{m}}$.

\section{LAGRANGE-TYPE INTERPOLATION SERIES}

In this section, we introduce the analytic Kramer kernels $K$ for which a nonorthogonal sampling theorem in $\mathscr{H}_{K}$ holds. We prove a converse result: From a sampling formula in $\mathscr{H}_{K}$ we deduce when $K$ is an analytic Kramer kernel. Finally, we prove the main result: a necessary and sufficient condition ensuring that the Kramer sampling result can be expressed as a Lagrange-type interpolation series.

\subsection{The Abstract Kramer Sampling Result}

Consider the data

$$
\left\{z_{n}\right\}_{n=1}^{\infty} \in \mathbb{C} \quad \text { and } \quad\left\{a_{n}\right\}_{n=1}^{\infty} \in \mathbb{C} \backslash\{0\} .
$$


Definition 2 (Analytic Kramer Kernel). An analytic kernel $K: \mathbb{C} \longrightarrow \mathscr{H}$ is said to be an analytic Kramer kernel (with respect to the data (7)) if it satisfies $K\left(z_{n}\right)=a_{n} x_{n}, n \in \mathbb{N}$, for some Riesz basis $\left\{x_{n}\right\}_{n=1}^{\infty}$ of $\mathscr{H}$.

A sequence $\left\{S_{n}\right\}_{n=1}^{\infty}$ of functions in the space $\mathscr{H}_{K}$ is said to have the interpolation property (with respect to the data (7)) if

$$
S_{n}\left(z_{m}\right)=a_{n} \delta_{n, m} .
$$

Thus, an analytic kernel $K$ is an analytic Kramer one if and only if the sequence of functions $\left\{S_{n}\right\}_{n=1}^{\infty}$ in $\mathscr{H}_{K}$ given by (5), where $\left\{y_{n}\right\}_{n=1}^{\infty}$ is the dual Riesz basis of $\left\{x_{n}\right\}_{n=1}^{\infty}$, has the interpolation property with respect to the same data (7).

Concerning the existence of analytic Kramer kernels, it has been proved in [11] that, associated with any arbitrary sequence of complex numbers $\left\{z_{n}\right\}_{n=1}^{\infty}$ such that $\lim _{n \rightarrow \infty}\left|z_{n}\right|=+\infty$, there exists an analytic Kramer kernel $K$.

Under the notation introduced so far an abstract version of the classical Kramer sampling theorem sampling [15] holds in $\mathscr{H}_{K}$; this is a slight modification of a sampling result in [14]. For notational purposes we include its proof.

Theorem 2 (Kramer Sampling Theorem). Let $K: \mathbb{C} \longrightarrow \mathscr{H}$ be an analytic Kramer kernel, and assume that the interpolation property (8) holds for some sequences $\left\{z_{n}\right\}_{n=1}^{\infty}$ in $\mathbb{C}$ and $\left\{a_{n}\right\}_{n=1}^{\infty}$ in $\mathbb{C} \backslash\{0\}$. Let $\mathscr{H}_{K}$ be the corresponding RKHS of entire functions. Then any $f \in \mathscr{H}_{K}$ can be recovered from its samples $\left\{f\left(z_{n}\right)\right\}_{n=1}^{\infty}$ by means of the sampling series

$$
f(z)=\sum_{n=1}^{\infty} f\left(z_{n}\right) \frac{S_{n}(z)}{a_{n}}, \quad z \in \mathbb{C},
$$

where the reconstruction functions $S_{n}$ are given in (5). The series converges absolutely and uniformly on compact subsets of $\mathbb{C}$.

Proof. First, notice that $\lim _{n \rightarrow \infty}\left|z_{n}\right|=+\infty$; otherwise the sequence $\left\{z_{n}\right\}_{n=1}^{\infty}$ contains a bounded subsequence and, hence, the entire function $S_{n} \equiv 0$ for all $n \in \mathbb{N}$, which contradicts (8). The anti-linear mapping $\mathscr{T}$ given by (4) is a bijective isometry between $\mathscr{H}_{\text {and }} \mathscr{H}_{K}$. As a consequence, the functions $\left\{S_{n}=\mathscr{T}\left(y_{n}\right)\right\}_{n=1}^{\infty}$ form a Riesz basis for $\mathscr{H}_{K}$; let $\left\{T_{n}\right\}_{n=1}^{\infty}$ be its dual Riesz basis. Expanding any $f \in \mathscr{H}_{K}$ in this basis we obtain

$$
f(z)=\sum_{n=1}^{\infty}\left\langle f, T_{n}\right\rangle_{\mathscr{H}_{K}} S_{n}(z) .
$$


Moreover,

$$
\left\langle f, T_{n}\right\rangle_{\mathscr{H}_{K}}=\overline{\left\langle x, x_{n}\right\rangle_{\mathscr{H}}}=\left\langle\frac{K\left(z_{n}\right)}{a_{n}}, x\right\rangle_{\mathscr{H}}=\frac{f\left(z_{n}\right)}{a_{n}} .
$$

Since a Riesz basis is an unconditional basis, the sampling series will be pointwise unconditionally convergent and hence, absolutely convergent. The uniform convergence is a standard result in the setting of the RKHS theory since $z \mapsto\|K(z)\|_{\mathscr{z}}$ is bounded on compact subsets of $\mathbb{C}$.

Riesz bases theory (see, e.g., [20]) assures the existence of two positive constants $0<A \leq B$ such that

$$
A\|f\|_{\mathscr{H}_{K}}^{2} \leq \sum_{n=1}^{\infty}\left|f\left(z_{n}\right) / a_{n}\right|^{2} \leq B\|f\|_{\mathscr{H}_{K}}^{2} \quad \text { for all } f \in \mathscr{H}_{K},
$$

that is, $\|f\|_{s}:=\left(\sum_{n=1}^{\infty}\left|f\left(z_{n}\right) / a_{n}\right|^{2}\right)^{1 / 2}$ defines an equivalent norm in $\mathscr{H}_{K}$. Following [12], we can say that the data (7) is a sampling set for $\mathscr{H}_{K}$; here the sequence of samples belongs to a weighted $\ell^{2}$ space. In [12], the authors characterize the reproducing kernel Hilbert spaces having a fixed sampling set.

The Whittaker-Shannon-Kotel'nikov sampling formula in $P W_{\pi}$ becomes a particular case of formula (9) in Theorem 2. Indeed, any $f \in P W_{\pi}$ can be written as

$$
f(z)=\frac{1}{\sqrt{2 \pi}} \int_{-\pi}^{\pi} \hat{f}(w) \mathrm{e}^{i z w} d w=\left\langle\frac{\mathrm{e}^{i z w}}{\sqrt{2 \pi}}, \overline{\hat{f}}\right\rangle_{L^{2}[-\pi, \pi]}, \quad z \in \mathbb{C} .
$$

The Fourier kernel $K(z):=\frac{\mathrm{e}^{\mathrm{i} z}}{\sqrt{2 \pi}} \in L^{2}[-\pi, \pi]$ is an analytic Kramer kernel for the data $\left\{z_{n}=n\right\}_{n \in \mathbb{Z}}$ and $\left\{a_{n}=1\right\}_{n \in \mathbb{Z}}$. In this case, as $\left\{\mathrm{e}^{\mathrm{i} n w} / \sqrt{2 \pi}\right\}_{n \in \mathbb{Z}}$ is an orthonormal basis for $L^{2}[-\pi, \pi]$ we get

$$
S_{n}(z)=\frac{1}{2 \pi}\left\langle\mathrm{e}^{\mathrm{i} z \cdot}, \mathrm{e}^{\mathrm{i} n \cdot}\right\rangle_{L^{2}[-\pi, \pi]}=\frac{\sin \pi(z-n)}{\pi(z-n)}, \quad z \in \mathbb{C} .
$$

As a consequence, we obtain the WSK sampling formula in $P W_{\pi}$ :

$$
f(z)=\sum_{n=-\infty}^{\infty} f(n) \frac{\sin \pi(z-n)}{\pi(z-n)}, \quad z \in \mathbb{C}
$$

The series converges absolutely and uniformly on horizontal strips of the complex plane.

It is worth to remark that a kernel $K$ can be an analytic Kramer kernel with respect to different data (7). For instance, the Fourier kernel is also 
an analytic Kramer kernel with respect to the data $\left\{z_{n}=n+\alpha\right\}_{n \in \mathbb{Z}}$ where $\alpha \in \mathbb{R}$ and $\left\{a_{n}=1\right\}_{n \in \mathbb{Z}}$. More generally, it is an analytic Kramer kernel with respect to any data $\left\{t_{n}\right\}_{n \in \mathbb{Z}} \subset \mathbb{R}$ and $\left\{a_{n}=1\right\}_{n \in \mathbb{Z}}$, where the points $t_{n}$ satisfy Kadec's condition $\sup _{n}\left|t_{n}-n\right|<1 / 4$ since the sequence $\left\{\mathrm{e}^{\mathrm{i} t_{n} w} / \sqrt{2 \pi}\right\}_{n \in \mathbb{Z}}$ is a Riesz basis for $L^{2}[-\pi, \pi]$ [20, p. 42].

\subsection{A Converse Result}

An interesting converse problem is to decide whether a sampling formula as (9), pointwise convergent in $\mathscr{H}_{K}$, implies the Kramer kernel condition in definition 2 for $K$. From formula (9) in Theorem 2 we derive that:

- From (5), for each $z \in \mathbb{C}$, the sequence $\left\{S_{n}(z)\right\}_{n=1}^{\infty} \in \ell^{2}(\mathbb{N})$.

- The sequence $\left\{f\left(z_{n}\right) / a_{n}\right\}_{n=1}^{\infty}$ belongs to $\ell^{2}(\mathbb{N})$ for any $f \in \mathscr{H}_{K}$, and

- $\sum_{n=1}^{\infty} \alpha_{n} S_{n}(z)=0$ for all $z \in \mathbb{C}$ and $\left\{\alpha_{n}\right\}_{n=1}^{\infty} \in \ell^{2}(\mathbb{N})$ implies $\alpha_{n}=0$ for all $n \in \mathbb{N}$, due to the uniqueness of a Riesz basis expansion in the RKHS $\mathscr{H}_{K}$.

It is worth to point out that these conditions are also sufficient to prove that $K$ is an analytic Kramer kernel.

Theorem 3. Let $\mathscr{H}_{K}$ be the range of a mapping $\mathscr{T}$ as in (4) considered as a RKHS with reproducing kernel $k(z, w)=\langle K(z), K(w)\rangle_{\mathscr{H}}$. Let $\left\{S_{n}\right\}_{n=1}^{\infty}$ be a sequence in $\mathscr{H}_{K}$ such that $\left\{S_{n}(z)\right\}_{n=1}^{\infty}$ belongs to $\ell^{2}(\mathbb{N})$ for each $z \in \mathbb{C}$. Suppose that the following conditions are fulfilled:

(i) $\sum_{n=1}^{\infty} \alpha_{n} S_{n}(z)=0$ for all $z \in \mathbb{C}$ and $\left\{\alpha_{n}\right\}_{n=1}^{\infty}$ in $\ell^{2}(\mathbb{N})$ implies $\alpha_{n}=0$ for
all $n$.

(ii) There exist sequences $\left\{z_{n}\right\}_{n=1}^{\infty}$ in $\mathbb{C}$ and $\left\{a_{n}\right\}_{n=1}^{\infty}$ in $\mathbb{C} \backslash\{0\}$ such that

$$
\left\{\frac{f\left(z_{n}\right)}{a_{n}}\right\}_{n=1}^{\infty} \in \ell^{2}(\mathbb{N}) \text { and } f(z)=\sum_{n=1}^{\infty} f\left(z_{n}\right) \frac{S_{n}(z)}{a_{n}}, \quad \text { for any } f \in \mathscr{H}_{K}
$$

where the sampling series is pointwise convergent in $\mathbb{C}$.

Then, the sequence $\left\{S_{n}\right\}_{n=1}^{\infty}$ is a Riesz basis for $\mathscr{H}_{K}$ and the kernel $K$ of the mapping $\mathscr{T}$ evaluated at $z \in \mathbb{C}$ can be expressed as $K(z)=\sum_{n=1}^{\infty} S_{n}(z) y_{n}$, where $\left\{y_{n}\right\}_{n=1}^{\infty}$ is the dual Riesz basis of the Riesz basis $\left\{x_{n}=\mathscr{T}^{-1}\left(S_{n}\right)\right\}_{n=1}^{\infty}$ in $\mathscr{H}$. In particular, $K\left(z_{n}\right)=a_{n} y_{n}$ for any $n \in \mathbb{N}$.

Proof. By defining $\tilde{k}(z, w):=\sum_{n=1}^{\infty} S_{n}(z) \overline{S_{n}(w)}$, we obtain a positive definite function which defines a RKHS $\mathscr{H}$, such that $\widetilde{\mathscr{H}} \subseteq \mathscr{H}_{K}$. Condition (i) implies that the sequence $\left\{S_{n}\right\}_{n=1}^{\infty}$ is an orthonormal basis for $\widetilde{\mathscr{H}}$ (see [17]). 
Now we prove that $\widetilde{\mathscr{H}}=\mathscr{H}_{K}$ and that the identity mapping $\widetilde{\mathscr{H}} \hookrightarrow \mathscr{H}_{K}$ is continuous. Take $f \in \mathscr{H}_{K}$, by condition ii), the sequence $\left\{f\left(z_{n}\right) a_{n}^{-1}\right\}_{n=1}^{\infty}$ is in $\ell^{2}(\mathbb{N})$. As a consequence, the series $\sum_{n=1}^{\infty} f\left(z_{n}\right) a_{n}^{-1} S_{n}$ converges in the norm of $\tilde{\mathscr{H}}$. By the reproducing kernel property, we have that the series $\sum_{n=1}^{\infty} f\left(z_{n}\right) a_{n}^{-1} S_{n}(z)$ is pointwise convergent. Comparing this with what we get from the sampling formula for $f$ we deduce that $f=\sum_{n=1}^{\infty} f\left(z_{n}\right) a_{n}^{-1} S_{n}$, where the convergence is in $\widetilde{\mathscr{H}}$ and, consequently, $f \in \tilde{\mathscr{H}}$.

Next we show the continuity of the identity mapping by applying the closed graph theorem. Indeed, let $\left\{f_{n}\right\}_{n=1}^{\infty}$ be a sequence such that $f_{n} \rightarrow f$ in $\widetilde{H}$ and $f_{n} \rightarrow g$ in $\mathscr{H}_{K}$ as $n \rightarrow \infty$. Using the reproducing property in both $\mathscr{H}_{K}$ and $\widetilde{H}$, for $z \in \mathbb{C}$ we have

$$
\begin{aligned}
\left|f_{n}(z)-f(z)\right| & \leq\left\|f_{n}-f\right\|_{\tilde{\mathscr{E}}} \sqrt{\tilde{k}(z, z)} \\
\left|f_{n}(z)-g(z)\right| & \leq\left\|f_{n}-g\right\|_{\mathscr{\mathscr { K }} K} \sqrt{k(z, z)} .
\end{aligned}
$$

Therefore, $\lim _{n \rightarrow \infty} f_{n}(z)=f(z)=g(z)$ for each $z \in \mathbb{C}$, and hence $f=g$.

Since it is also surjective, we infer that the norms $\|\cdot\|_{\mathscr{H}_{K}}$ and $\|\cdot\| \widetilde{\mathscr{C}}$ are equivalent from the open mapping theorem. As a consequence, the orthonormal basis $\left\{S_{n}\right\}_{n=1}^{\infty}$ in $\widetilde{\mathscr{H}}$ is a Riesz basis for $\mathscr{H}_{K}$.

Assuming that the mapping $\mathscr{T}$ is one-to-one, the sequence $\left\{x_{n}=\right.$ $\left.\mathscr{T}^{-1}\left(S_{n}\right)\right\}_{n=1}^{\infty}$ is a Riesz basis for $\mathscr{H}$; denote by $\left\{y_{n}\right\}_{n=1}^{\infty}$ its dual Riesz basis. Expanding $K(z)$ with respect to $\left\{y_{n}\right\}_{n=1}^{\infty}$, for each fixed $z \in \mathbb{C}$ we obtain

$$
K(z)=\sum_{n=1}^{\infty}\left\langle K(z), x_{n}\right\rangle_{\mathscr{H}} y_{n}=\sum_{n=1}^{\infty} S_{n}(z) y_{n}
$$

that is, the required expansion for $K(z)$.

Notice that the interpolatory condition $S_{n}\left(z_{m}\right)=a_{m} \delta_{n, m}$ comes out of a direct application of condition (ii) to $S_{n}$, followed by condition (i).

As to the case when, a priori, $\mathscr{T}$ is not known to be one-to-one, let $\left\{x_{n}\right\}_{n=1}^{\infty}$ be a sequence in $\mathscr{H}$ with $P\left(x_{n}\right) \neq 0$ for all $n$, where $P$ denotes the orthogonal projection onto the closed subspace $(\operatorname{Ker} \mathscr{T})^{\perp}$. Consider $S_{n}=\mathscr{T}\left(x_{n}\right) \in \mathscr{H}_{K}$, and suppose that these functions satisfy the hypotheses in Theorem 3. In this case, $\left\{S_{n}\right\}_{n=1}^{\infty}$ is a Riesz basis for $\mathscr{H}_{K}$. Consequently, since $S_{n}=\mathscr{T}\left[P\left(x_{n}\right)\right]$ and $\left.\mathscr{T}\right|_{P(\operatorname{Ker} \mathscr{T})}=0$, we obtain that $\left\{P\left(x_{n}\right)\right\}_{n=1}^{\infty}$ is a Riesz basis for $P(\mathscr{H})=(\operatorname{Ker} \mathscr{T})^{\perp}$. The result comes out taking into account the orthogonal sum $\mathscr{H}=(\operatorname{Ker} \mathscr{T})^{\perp} \oplus(\operatorname{Ker} \mathscr{T})$.

\subsection{Lagrange-Type Interpolation Series}

A more difficult question concerns whether the sampling expansion (9) can be written, in general, as a Lagrange-type interpolation series. 
For instance, for $f \in P W_{\pi}$ the WSK formula (12) can be written as the Lagrange-type interpolation series

$$
f(z)=\sum_{n=-\infty}^{\infty} f(n) \frac{P(z)}{(z-n) P^{\prime}(n)}, \quad z \in \mathbb{C},
$$

by taking $P(z)=(\sin \pi z) / \pi$, an entire function having only simple zeros at $\mathbb{Z}$.

The case where the sequence $\left\{x_{n}\right\}_{n=1}^{\infty}$ in Definition 2 is an orthonormal basis for $\mathscr{H}$ was studied in [7]: A necessary and suficient condition involves the ZR property. Next, we prove that the same necessary and sufficient condition holds in the general case of analytic Kramer kernels $K$ involving Riesz bases.

Theorem 4. Let $\mathscr{H}_{K}$ be a RKHS of entire functions obtained from an analytic Kramer kernel $K$ with respect to the data $\left\{z_{n}\right\}_{n=1}^{\infty} \subset \mathbb{C}$ and $\left\{a_{n}\right\}_{n=1}^{\infty} \in \mathbb{C} \backslash\{0\}$, that is, $K\left(z_{n}\right)=a_{n} x_{n}, n \in \mathbb{N}$, for some Riesz basis $\left\{x_{n}\right\}_{n=1}^{\infty}$ for $\mathscr{H}$. Then, the sampling formula (9) for $\mathscr{H}_{K}$ can be written as a Lagrange-type interpolation series

$$
f(z)=\sum_{n=1}^{\infty} f\left(z_{n}\right) \frac{P(z)}{\left(z-z_{n}\right) P^{\prime}\left(z_{n}\right)}, \quad z \in \mathbb{C},
$$

where $P$ denotes an entire function having only simple zeros at $\left\{z_{n}\right\}_{n=1}^{\infty}$ if and only if the space $\mathscr{H}_{K}$ satisfies the ZR property.

Proof. For the sufficient condition we have to prove that sampling formula (9) can be written as a Lagrange-type interpolation series (13) for some entire function $P$. First, we prove that the only zeros of the sampling function $S_{n}$ are given by $\left\{z_{r}\right\}_{r \neq n}$. Suppose that $S_{n}(w)=0$, then by hypothesis the function $S_{n}(z) /(z-w)$ is in $\mathscr{H}_{K}$. Hence, the function

$$
\frac{z-z_{n}}{z-w} S_{n}(z)=S_{n}(z)+\frac{w-z_{n}}{z-w} S_{n}(z)
$$

also belongs to $\mathscr{H}_{K}$. If $w \notin\left\{z_{r}\right\}_{r \neq n}$, the function $\frac{z-z_{n}}{z-w} S_{n}(z)$ in $\mathscr{H}_{K}$ vanishes at the sequence $\left\{z_{r}\right\}_{r=1}^{\infty}$ which implies that $S_{n} \equiv 0$, to give a contradiction. In addition, the zeros of $S_{n}$ are simple; indeed, suppose that $z_{m}$ is a multiple zero of $S_{n}$. Proceeding as above, the function $\frac{z-z_{n}}{z-z_{m}} S_{n}(z)$ belongs to $\mathscr{H}_{K}$ and vanishes at $\left\{z_{r}\right\}_{r=1}^{\infty}$ which again implies that $S_{n} \stackrel{z-z_{m}}{\equiv}$.

Consequently, choosing an entire function $Q$ having only simple zeros at $\left\{z_{n}\right\}_{n=1}^{\infty}$, for each $n \in \mathbb{N}$ there exists an entire function $A_{n}$ without zeros such that $\left(z-z_{n}\right) S_{n}(z)=Q(z) A_{n}(z), z \in \mathbb{C}$. Next, we prove that there exists an entire function $A$ without zeros and a sequence $\left\{\sigma_{n}\right\}_{n=1}^{\infty}$ in $\mathbb{C} \backslash\{0\}$ such 
that $A_{n}(z)=\sigma_{n} A(z)$ for all $z \in \mathbb{C}$. For $m \neq n$ the function $\frac{z-z_{n}}{z-z_{m}} S_{n}(z)$ in $\mathscr{H}_{K}$ has its zeros at $\left\{z_{r}\right\}_{r \neq m}$. Thus, the sampling formula (9) gives

$$
\frac{z-z_{n}}{z-z_{m}} S_{n}(z)=\left[\left(z_{m}-z_{n}\right) S_{n}^{\prime}\left(z_{m}\right)\right] \frac{S_{m}(z)}{a_{m}}, \quad z \in \mathbb{C} .
$$

Fixing $m=1$, we conclude that $A_{n}(z)=\sigma_{n} A(z)$ where $A=A_{1}$ and $\sigma_{n}=$ $\left(z_{1}-z_{n}\right) S_{n}^{\prime}\left(z_{1}\right) \neq 0$ for $n \in \mathbb{N} \backslash\{1\}$ and $\sigma_{1}=1$. Hence, $S_{n}(z)=\frac{\sigma_{n} Q(z) A(z)}{z-z_{n}}$ for $z \neq z_{n}$ and $S_{n}\left(z_{n}\right)=a_{n}=\sigma_{n} Q^{\prime}\left(z_{n}\right) A\left(z_{n}\right)$. Substituting in (9) we obtain the Lagrange-type interpolation series (13) where $P(z)=A(z) Q(z)$.

For the necessary condition, assume that the sampling formula in $\mathscr{H}_{K}$ takes the form of a Lagrange-type interpolation series (13). Given $g \in \mathscr{H}_{K}$, there exists $x \in \mathscr{H}$ such that $g(z)=\langle K(z), x\rangle, z \in \mathbb{C}$. Assuming that $g(w)=$ 0 , we have to prove that the function $g(z) /(z-w)$ belongs to $\mathscr{H}_{K}$. The sampling expansion for $g$ at $w$ gives

$$
\sum_{n=1}^{\infty} g\left(z_{n}\right) \frac{P(w)}{\left(w-z_{n}\right) P^{\prime}\left(z_{n}\right)}=0 \text {. }
$$

We distinguish two cases:

(i) $w \in \mathbb{C} \backslash\left\{z_{n}\right\}_{n=1}^{\infty}$. As $P(w) \neq 0$, from (14) we obtain

$$
\sum_{n=1}^{\infty} g\left(z_{n}\right) \frac{1}{\left(w-z_{n}\right) P^{\prime}\left(z_{n}\right)}=0 .
$$

Thus,

$$
\begin{aligned}
g(z) & =\sum_{n=1}^{\infty} g\left(z_{n}\right) \frac{P(z)}{\left(z-z_{n}\right) P^{\prime}\left(z_{n}\right)}-\sum_{n=1}^{\infty} g\left(z_{n}\right) \frac{P(z)}{\left(w-z_{n}\right) P^{\prime}\left(z_{n}\right)} \\
& =(z-w) \sum_{n=1}^{\infty} g\left(z_{n}\right) \frac{P(z)}{P^{\prime}\left(z_{n}\right)} \frac{1}{\left(z-z_{n}\right)\left(z_{n}-w\right)} .
\end{aligned}
$$

Therefore, the entire function $G(z):=g(z) /(z-w)$ can be recovered from its samples at $\left\{z_{n}\right\}_{n=1}^{\infty}$ through the formula

$$
G(z)=\sum_{n=1}^{\infty} G\left(z_{n}\right) \frac{P(z)}{\left(z-z_{n}\right) P^{\prime}\left(z_{n}\right)}, \quad z \in \mathbb{C} .
$$

Moreover, the function $G$ is in $\mathscr{H}_{K}$ because $G(z)=\langle K(z), y\rangle_{\mathscr{H}}$, where $y \in$ $\mathscr{H}$ has the expansion $y=\sum_{n=1}^{\infty}\left\langle y, x_{n}\right\rangle y_{n}$ with respect to the dual Riesz basis 
$\left\{y_{n}\right\}_{n=1}^{\infty}$ of $\left\{x_{n}\right\}_{n=1}^{\infty}$, where the coefficients are given by

$$
\left\{\left\langle y, x_{n}\right\rangle:=\frac{1}{\bar{z}_{n}-\bar{w}}\left\langle x, x_{n}\right\rangle\right\}_{n=1}^{\infty} \in \ell^{2}(\mathbb{N})
$$

Indeed, sampling formula (13) for $S_{n}$ gives $S_{n}(z)=a_{n} \frac{P(z)}{\left(z-z_{n}\right) P^{\prime}\left(z_{n}\right)}$. Hence, by using the biorthogonality $\left\langle x_{n}, y_{n}\right\rangle=\delta_{n, m}$, we obtain

$$
\langle K(z), y\rangle=\sum_{n=1}^{\infty} \frac{S_{n}(z) \overline{\left\langle x, x_{n}\right\rangle}}{w-z_{n}}=G(z), \quad z \in \mathbb{C},
$$

where we have used (15), and the result that $\overline{\left\langle x, x_{n}\right\rangle}=g\left(z_{n}\right) / a_{n}, n \in \mathbb{N}$.

(ii) $w=z_{m}$ for some $m \in \mathbb{N}$. As $g\left(z_{m}\right)=0$, the sampling expansion for $g$ reads

$$
g(z)=\sum_{\substack{n=1 \\ n \neq m}}^{\infty} g\left(z_{n}\right) \frac{P(z)}{\left(z-z_{n}\right) P^{\prime}\left(z_{n}\right)}, \quad z \in \mathbb{C} .
$$

Setting $P(z)=\left(z-z_{m}\right) Q_{m}(z)$ we have $P^{\prime}(z)=Q_{m}(z)+\left(z-z_{m}\right) Q_{m}^{\prime}(z)$ and, hence,

$$
P^{\prime}\left(z_{k}\right)= \begin{cases}\left(z_{k}-z_{m}\right) Q_{m}^{\prime}\left(z_{k}\right) & \text { if } k \neq m \\ Q_{m}\left(z_{m}\right) & \text { if } k=m\end{cases}
$$

Hence,

$$
\frac{g(z)}{z-z_{m}}=\sum_{\substack{n=1 \\ n \neq m}}^{\infty} \frac{g\left(z_{n}\right)}{z_{n}-z_{m}} \frac{Q_{m}(z)}{\left(z-z_{n}\right) Q_{m}^{\prime}\left(z_{n}\right)}, \quad z \in \mathbb{C}
$$

Using the uniform convergence of the series in (16) we deduce that this series defines a continuous function. Hence, taking the limit as $z \rightarrow z_{m}$ we obtain

$$
g^{\prime}\left(z_{m}\right)=\sum_{\substack{n=1 \\ n \neq m}}^{\infty} \frac{g\left(z_{n}\right)}{z_{n}-z_{m}} \frac{Q_{m}\left(z_{m}\right)}{\left(z_{m}-z_{n}\right) Q_{m}^{\prime}\left(z_{m}\right)}
$$

Now we prove that

$$
\frac{g(z)}{z-z_{m}}=\sum_{\substack{n=1 \\ n \neq m}}^{\infty} \frac{g\left(z_{n}\right)}{z_{n}-z_{m}} \frac{P(z)}{\left(z-z_{n}\right) P^{\prime}\left(z_{n}\right)}+g^{\prime}\left(z_{m}\right) \frac{P(z)}{\left(z-z_{m}\right) P^{\prime}\left(z_{m}\right)} .
$$


Indeed, substituting (17) into (18) we obtain

$$
\begin{aligned}
\sum_{\substack{n=1 \\
n \neq m}}^{\infty} & {\left[\frac{g\left(z_{n}\right)}{z_{n}-z_{m}} \frac{P(z)}{\left(z-z_{n}\right) P^{\prime}\left(z_{n}\right)}+\frac{g\left(z_{m}\right)}{z_{n}-z_{m}} \frac{Q_{m}(z)}{\left(z_{m}-z_{n}\right) Q_{m}^{\prime}\left(z_{n}\right)}\right] } \\
& =\sum_{\substack{n=1 \\
n \neq m}}^{\infty} \frac{g\left(z_{n}\right)}{z_{n}-z_{m}} \frac{Q_{m}(z)}{Q_{m}^{\prime}\left(z_{n}\right)}\left[\frac{z-z_{m}}{\left(z_{n}-z_{m}\right)\left(z-z_{n}\right)}-\frac{1}{z_{n}-z_{m}}\right] \\
& =\sum_{\substack{n=1 \\
n \neq m}}^{\infty} \frac{g\left(z_{n}\right)}{z_{n}-z_{m}} \frac{Q_{m}(z)}{\left(z-z_{n}\right) Q_{m}^{\prime}\left(z_{n}\right)} \\
& =\frac{g(z)}{z-z_{m}} .
\end{aligned}
$$

Thus, defining $y \in \mathscr{H}$ by the expansion $y=\sum_{n=1}^{\infty}\left\langle y, x_{n}\right\rangle y_{n}$ where the coefficients $\left\{\left\langle y, x_{n}\right\rangle\right\}_{n=1}^{\infty}$ in $\ell^{2}(\mathbb{N})$ are given by

$$
\left\langle y, x_{n}\right\rangle:= \begin{cases}\frac{\left\langle x, x_{n}\right\rangle}{\bar{z}_{n}-\bar{z}_{m}} & \text { if } n \neq m \\ \frac{g^{\prime}\left(z_{m}\right)}{a_{m}} & \text { if } n=m\end{cases}
$$

and proceeding as in case (i), it may be shown that

$$
\frac{g(z)}{z-z_{m}}=\langle K(z), y\rangle, \quad z \in \mathbb{C},
$$

which proves that the function $g(z) /\left(z-z_{m}\right)$ belongs to $\mathscr{H}_{K}$. This concludes the proof of the theorem.

Some comments concerning Theorem 4 are in order:

1. In the proof of Theorem 4 we have found that the entire function $P$ satisfies:

$$
\left(z-z_{n}\right) S_{n}(z)=\sigma_{n} P(z), \quad z \in \mathbb{C}
$$

for some sequence $\left\{\sigma_{n}\right\}_{n=1}^{\infty} \in \mathbb{C} \backslash\{0\}$. In the case where $P$ can be factorized as $P(z)=A(z) Q(z)$, where $Q$ denotes a canonical product having its simple zeros at $\left\{z_{n}\right\}_{n=1}^{\infty}$ and $A$ is an entire function 
without zeros, then the Lagrange-type interpolation series (13) can be expressed as

$$
f(z)=\sum_{n=1}^{\infty} f\left(z_{n}\right) \frac{A(z)}{A\left(z_{n}\right)} \frac{Q(z)}{\left(z-z_{n}\right) Q^{\prime}\left(z_{n}\right)}, \quad z \in \mathbb{C} .
$$

2. In particular, as de Branges space satisfy the ZR property the orthogonal sampling formulas in these spaces, first proved in [16], can be expressed as Lagrange-type interpolation series (see [11] for some nontrivial examples).

3. It is worth to mention that if one particular sampling formula (9) can be written as a Lagrange-type interpolation formula, then the same occurs for all the sampling formulas (9) obtained from other compatible data (7). Besides, if the space $\mathscr{H}_{K}$ does not satisfy the ZR property, we conclude that it does not exist any data (7) for which the kernel $K$ is an analytic Kramer kernel and the associated sampling formula (9) can be written as a Lagrange-type interpolation series.

\subsection{Some Illustrative Examples}

Closing the article, we show some examples illustrating Theorems 2 and 4 .

\subsubsection{Classical Polynomial Interpolation}

Let $\mathscr{P}_{N}(\mathbb{C})$ be the set of polynomials with complex coefficients of degree less or equal $N$. As we proved in Theorem $1, \mathscr{P}_{N}(\mathbb{C})$ coincides with the corresponding $\mathscr{H}_{K}$ space where $K(z):=\sum_{n=0}^{N} \mathbf{p}_{\mathbf{n}} z^{n}$ being $\left\{\mathbf{p}_{0}, \mathbf{p}_{1}, \ldots, \mathbf{p}_{\mathrm{N}}\right\}$ any basis for the euclidean space $\mathscr{H}:=\mathbb{C}^{N+1}$. Consider $N+1$ different points $\left\{z_{n}\right\}_{n=0}^{N}$ in $\mathbb{C}$; it is easy to prove that $K$ is an analytic Kramer kernel with respect the data $\left\{z_{n}\right\}_{n=0}^{N}$ and $\left\{a_{n}=1\right\}_{n=0}^{N}$. Indeed, the set $\left\{K\left(z_{n}\right)=\mathbf{q}_{\mathbf{n}}\right\}_{n=0}^{N}$ is linearly independent in $\mathbb{C}^{N+1}$ by using Vandermonde determinants, that is, it forms a (Riesz) basis for $\mathbb{C}^{N+1}$. Thus, Theorems 2 and 4 give, for any $f \in \mathscr{P}_{N}(\mathbb{C})$

$$
f(z)=\sum_{n=0}^{N} f\left(z_{n}\right) S_{n}(z)=\sum_{n=0}^{N} f\left(z_{n}\right) \frac{P(z)}{\left(z-z_{n}\right) P^{\prime}\left(z_{n}\right)}, \quad z \in \mathbb{C},
$$

where $S_{n}(z)=\left\langle K(z), \mathbf{q}_{\mathbf{n}}^{*}\right\rangle$, being $\left\{\mathbf{q}_{\mathbf{n}}^{*}\right\}_{n=0}^{N}$ the dual basis of $\left\{\mathbf{q}_{\mathbf{n}}\right\}_{n=0}^{N}$ in $\mathbb{C}^{N+1}$, and $P(z)=\prod_{n=0}^{N}\left(z-z_{n}\right)$. 


\subsubsection{The Paley-Wiener-Levinson Theorem Revisited}

Let $\left\{z_{n}\right\}_{n \in \mathbb{Z}}$ be a sequence in $\mathbb{C}$ for which $\sup _{n}\left|\operatorname{Re} z_{n}-n\right|<1 / 4$ and $\sup _{n}\left|\operatorname{Im} z_{n}\right|<\infty$. It is known that the system $\left\{\mathrm{e}^{i z_{n} w} / \sqrt{2 \pi}\right\}_{n \in \mathbb{Z}}$ is a Riesz basis for $L^{2}[-\pi, \pi]$ (see [20, p. 196]). The Fourier kernel $K(z)=\frac{\mathrm{e}^{i z}}{\sqrt{2 \pi}} \in L^{2}[-\pi, \pi]$ is an analytic Kramer kernel for the data $\left\{z_{n}\right\}_{n \in \mathbb{Z}}$ and $\left\{a_{n}=1\right\}_{n \in \mathbb{Z}}$. Thus, Theorems 2 and 4 give, for any $f \in P W_{\pi}$

$$
f(z)=\sum_{n=-\infty}^{\infty} f\left(z_{n}\right) S_{n}(z)=\sum_{n=-\infty}^{\infty} f\left(z_{n}\right) \frac{P(z)}{\left(z-z_{n}\right) P^{\prime}\left(z_{n}\right)}, \quad z \in \mathbb{C},
$$

where, for $n \in \mathbb{Z}$, the sampling function $S_{n}(z)=\left\langle K(z), h_{n}\right\rangle_{L^{2}[-\pi, \pi]}$, being $\left\{h_{n}(w)\right\}_{n \in \mathbb{Z}}$ the dual Riesz basis of $\left\{\mathrm{e}^{i z_{n} w} / \sqrt{2 \pi}\right\}_{n \in \mathbb{Z}}$ in $L^{2}[-\pi, \pi]$, and $P$ is the entire function having only simple zeros at $\left\{z_{n}\right\}_{n \in \mathbb{Z}}$. Since a result from Titchmarsh [18] assures that the functions in $P W_{\pi}$ are completely determined by their zeros, we derive that, up to a constant factor, the entire function $P$ coincides with the infinite product

$$
\left(z-z_{0}\right) \prod_{n=1}^{\infty}\left(1-\frac{z}{z_{n}}\right)\left(1-\frac{z}{z_{-n}}\right)
$$

Indeed, the function $S_{0} \in P W_{\pi}$ has only simple zeros at $\left\{z_{m}\right\}_{m \neq 0}\left(S_{0}\left(z_{m}\right)=\right.$ $\left.\delta_{0, m}\right)$. Suppose on the contrary that $s \notin\left\{z_{m}\right\}_{m \neq 0}$ is a zero of $S_{0}$. According to the classical Paley-Wiener theorem, the function $S(z):=\left(z-z_{0}\right) S_{0}(z) /(z-$ s) belongs to $P W_{\pi}$ and vanishes at every $z_{n}$. If we take into account the completeness of the Riesz basis $\left\{\mathrm{e}^{i z_{n} w} / \sqrt{2 \pi}\right\}_{n \in \mathbb{Z}}$, this implies that $S \equiv 0$, a contradiction. Therefore, by using the Titchmarsh's result, the function $S_{0}$ coincides, up to a constant factor, with the (convergent) product $\prod_{n=1}^{\infty}(1-$ $\left.\frac{z}{z_{n}}\right)\left(1-\frac{z}{z_{-n}}\right)$. Since Theorem 4 gives $\left(z-z_{n}\right) S_{n}(z)=\sigma_{n} P(z)$ for all $n \in \mathbb{Z}$, we obtain the desired result.

\subsubsection{Finite Cosine Transform}

It is known that any function $f(z)=\langle\cos z x, F(x)\rangle_{L^{2}[0, \pi]}, z \in \mathbb{C}$, where $F \in L^{2}[0, \pi]$, can be expanded as the sampling formula $[13$, p. 5$]$

$$
f(z)=f(0) \frac{\sin \pi z}{\pi z}+\frac{2}{\pi} \sum_{n=0}^{\infty} f(n) \frac{(-1)^{n} z \sin \pi z}{z^{2}-n^{2}}, \quad z \in \mathbb{C} .
$$

This sampling formula cannot be expressed as a Lagrange-type interpolation series since, as we noticed in section 3, the corresponding $\mathscr{H}_{K}$ space does not satisfy the ZR property. 


\subsubsection{An Example Involving a Sobolev Space}

Finally, we give an example taken from [10] of a RKHS $\mathscr{H}_{K}$, built from the Sobolev Hilbert space $\mathscr{H}:=H^{1}(-\pi, \pi)$, where the ZR property fails. Namely, consider the Sobolev Hilbert space $H^{1}(-\pi, \pi)$ with its usual inner product

$$
\langle f, g\rangle_{1}=\int_{-\pi}^{\pi} f(x) \overline{g(x)} d x+\int_{-\pi}^{\pi} f^{\prime}(x) \overline{g^{\prime}(x)} d x, \quad f, g \in H^{1}(-\pi, \pi) .
$$

The sequence $\left\{e^{i n x}\right\}_{n \in \mathbb{Z}} \cup\{\sinh x\}$ forms an orthogonal basis for $H^{1}(-\pi, \pi)$ : It is straightforward to prove that the orthogonal complement of $\left\{e^{i n x}\right\}_{n \in \mathbb{Z}}$ in $H^{1}(-\pi, \pi)$ is a one-dimensional space for which $\sinh x$ is a basis. For a fixed $a \in \mathbb{C} \backslash \mathbb{Z}$ we define a kernel

$$
\begin{aligned}
K_{a}: \mathbb{C} & \longrightarrow H^{1}(-\pi, \pi) \\
& \longrightarrow K_{a}(z),
\end{aligned}
$$

by setting

$$
\left[K_{a}(z)\right](x)=(z-a) e^{i z x}+\sin \pi z \sinh x, \quad \text { for } x \in(-\pi, \pi) .
$$

Clearly, $K_{a}$ defines an analytic Kramer kernel. Expanding $K_{a}(z) \in$ $H^{1}(-\pi, \pi)$ in the former orthogonal basis we obtain

$$
K_{a}(z)=[1-i(z-a)] \sin \pi z \sinh x+(z-a) \sum_{n=-\infty}^{\infty} \frac{1+z n}{1+n^{2}} \operatorname{sinc}(z-n) e^{i n x} .
$$

As a consequence, Theorem 2 gives the following sampling result in $\mathscr{H}_{K_{a}}$; Any function $f \in \mathscr{H}_{K_{a}}$ can be recovered from its samples $\{f(a)\} \cup\{f(n)\}_{n \in \mathbb{Z}}$ by means of the sampling formula

$$
f(z)=[1-i(z-a)] \frac{\sin \pi z}{\sin \pi a} f(a)+\sum_{n=-\infty}^{\infty} f(n) \frac{z-a}{n-a} \frac{1+z n}{1+n^{2}} \operatorname{sinc}(z-n) .
$$

The function $(z-a) \operatorname{sinc} z$ belongs to $\mathscr{H}_{K_{a}}$ since $(z-a) \operatorname{sinc} z=$ $\left\langle K_{a}(z), 1 / 2 \pi\right\rangle_{1}$ for all $z \in \mathbb{C}$. However, by using the sampling formula for $\mathscr{H}_{K_{a}}$ it is straightforward to check that the function sinc $z$ does not belong to $\mathscr{H}_{K_{a}}$; as a consequence, the above sampling formula cannot be expressed as a Lagrange-type interpolation series.

\section{ACKNOWLEDGMENTS}

This work has been supported by the grant MTM2009-08345 from the Spanish Ministerio de Ciencia e Innovación (MICINN). 


\section{REFERENCES}

1. M.H. Annaby and G. Freiling (2000). Sampling expansions associated with Kamke problems. Math. Z. 234: 163-189.

2. L. de Branges (1986). Hilbert Spaces of Entire Functions. Prentice-Hall, Englewood Cliffs, NJ.

3. P. Butzer, J.R. Higgins, and R.L. Stens (2000). Sampling theory in signal analysis. In: Development of Mathematics 1950-2000 (J.P. Pier, ed.). Birkhäuser, Basel, pp. 193-234.

4. P. Butzer and G. Nasri-Roudsari (1997). Kramer's sampling theorem in signal analysis and its role in mathematics. In: Image Processing; Mathematical Methods and Applications. Proeedings of the IMA Conference, Cranfield University, UK (J.M. Blackledge, ed.). Clarendon Press, Oxford, pp. 49-95.

5. W.N. Everitt and G. Nasri-Roudsari (1999). Interpolation and sampling theories, and linear ordinary boundary value problems. In: Sampling Theory in Fourier and Signal Analysis: Advanced Topics (J.R. Higgins and R.L. Stens, eds.). Oxford University Press, Oxford, pp. 96-129.

6. W.N. Everitt, G. Nasri-Roudsari, and J. Rehberg (1998). A note on the analytic form of the Kramer sampling theorem. Results Math. 34:310-319.

7. W.N. Everitt, A.G. García, and M.A. Hernández-Medina (2008). On Lagrange-type interpolation series and analytic Kramer kernels. Results Math. 51:215-228.

8. A.G. García and M.A. Hernández-Medina (2001). The discrete Kramer sampling theorem and indeterminate moment problems. J. Comp. Appl. Math. 134:13-22.

9. A.G. García and M.A. Hernández-Medina (2003). Discrete Sturm-Liouville problems, Jacobi matrices and Lagrange-type interpolation series. J. Math. Anal. Appl. 280:221-231.

10. A.G. García and L.L. Littlejohn (2004). On analytic sampling theory. J. Comp. Appl. Math. $171: 235-246$.

11. A.G. García, M.A. Hernández-Medina, and F.H. Szafraniec (2011) Analytic Kramer kernels, Lagrange-type interpolation series and de Branges spaces. Complex Variables and Elliptic Equations. DOI: $10.1080 / 17476933.2010 .551206$

12. D. Han, M.Z. Nashed, and Q. Sun (2009). Sampling expansions in reproducing kernel Hilbert and Banach spaces. Numer. Funct. Anal. Optim. 30:971-987.

13. J.R. Higgins (1996). Sampling Theory in Fourier and Signal Analysis: Foundations. Oxford University Press, Oxford, UK.

14. J.R. Higgins (2001). A sampling principle associated with Saitoh's fundamental theory of linear transformations. In: Analytic Extension Formulas and Their Applications (S. Saitoh, N. Hayashi, and M. Yamamoto, eds.). Kluwer Academic, Dordrecht, pp. 73-86.

15. H.P. Kramer (1957). A generalized sampling theorem. J. Math. Phys. 63:68-72.

16. M.Z. Nashed and G.G. Walter (1991). General sampling theorems for functions in reproducing kernel spaces. Math. Control Signals Systems 4:363-390.

17. F. H. Szafraniec (2000). The reproducing kernel Hilbert space and its multiplication operators. Operator Th. Adv. Appl. 114:253-263.

18. E. C. Titchmarsh (1926). The zeros of certain integral functions. Proc. London Math. Soc. 25:283-302.

19. A.E. Taylor and D.C. Lay (1980). Introduction to Functional Analysis. John Wiley \& Sons, New York.

20. R.M. Young (1980). An Introduction to Nonharmonic Fourier Series. Academic Press, New York.

21. A.I. Zayed (1993). Advances in Shannon's Sampling Theory. CRC Press, Boca Raton, FL. 